\title{
Outer Synchronization of Complex Networks with Nondelayed and Time-Varying Delayed Couplings via Pinning Control or Impulsive Control
}

\author{
Jianwen Feng, ${ }^{1}$ Sa Sheng, ${ }^{1}$ Ze Tang, ${ }^{2}$ and Yi Zhao' \\ ${ }^{1}$ College of Mathematics and Computational Sciences, Shenzhen University, Shenzhen 518060, China \\ ${ }^{2}$ Department of Electrical Engineering, Yeungnam University, Gyeongsan, Gyeongbuk 712-749, Republic of Korea \\ Correspondence should be addressed to Jianwen Feng; fengjw@szu.edu.cn
}

Received 9 July 2014; Accepted 15 August 2014

Academic Editor: Sakthivel Rathinasamy

Copyright (C) 2015 Jianwen Feng et al. This is an open access article distributed under the Creative Commons Attribution License, which permits unrestricted use, distribution, and reproduction in any medium, provided the original work is properly cited.

\begin{abstract}
The outer synchronization problem between two complex networks with nondelayed and time-varying delayed couplings via two different control schemes, namely, pinning control and impulsive control, is considered. Firstly, by applying pinning control to a fraction of the network nodes and using a suitable Lyapunov function, we obtain some new and useful synchronization criteria, which guarantee the outer synchronization between two complex networks. Secondly, impulsive control is added to the nodes of corresponding response network. Based on the generalized inequality about time-varying delayed different equation, the sufficient conditions for outer synchronization are derived. Finally, some examples are presented to demonstrate the effectiveness and feasibility of the results obtained in this paper.
\end{abstract}

\section{Introduction}

As an important and typical collective behavior of complex networks, synchronization has been studied extensively in various research fields since it has been observed widely in potential applications in many different areas such as population dynamic, power system, chemical process simulation, and automatic control [1-7]. In real worlds, a great number of complex networks cannot achieve synchronization by themselves. Therefore, many kinds of effective control methods, for instance, adaptive control [8,9], pinning control [10-13], impulsive control [14], and so forth, have been developed to drive complex networks to reach synchronization. Some control schemes based on imposing the controllers on all the nodes in the networks, which is difficult to implement and impractical for some large scale networks. Considering these drawbacks, pinning control is proposed as a powerful technique because it is effective and relatively easily realized by controlling a small percentage of the nodes instead of all the nodes in whole network. As a result, some authors have devoted themselves to investigating different pinning control schemes for various complex dynamical networks. Chen et al. [10] investigated both specific and random pinning schemes for linearly and diffusively coupled networks. Xiang and Zhu proved that a single controller can pin a coupled complex network to homogeneous solutions in [11]. Compared with existing continuous or discrete pinning control schemes, impulsive control, another type of control methods, has attracted lots of researchers because of its potential advantage over general continuous control schemes [14-17]. For instance, Lu et al. in [15] proposed a new approach for analyzing pinning stability in a complex dynamical network via impulsive control. In [16], the authors addressed a directed dynamical network with impulsive coupling by a single impulsive controller.

It should be noted that the aforementioned works on network synchronization have mainly focused on analyzing the synchronization behavior within a network, which is regarded as "inner synchronization." In fact, there is another type of network synchronization for networks, which is known as "outer synchronization." Generally speaking, it is a type of synchronization between two or more coupled networks [14, 18-22], which means the corresponding nodes of coupled networks will achieve synchronization regardless 
of synchronization of the inner networks. A typical example is the acquired immune deficiency syndrome, AIDS for brief, which originally outbroke among gorillas and afterwards was contagious to human beings unexpectedly, where cows groups and human beings could be regarded as two different networks in terms of network language. In recent years, some researchers began to put their interests on synchronization between two coupled complex networks. In [18], the authors first analyzed synchronization between two interacting populations of different phase oscillators. In [20], Sun et al. proved that outer synchronization can be asymptotically reached by using arbitrary coupling strength for two networks with balanced structure topology. Moreover, mixed outer synchronization between two complex dynamical networks with nonidentical nodes and output coupling is investigated via impulsive hybrid control in [23]. However, these research works exclude time-delay couplings, which cannot be ignored since they are ubiquitous in the real world. Considering these reasons, Zheng and Shao discussed the inner-outer synchronization between two complex networks with nondelayed and delayed coupling in [24] by applying the pinning control method. It should be mentioned that the time delay in [24] is fixed. For the varying time delay, in [22], Zheng investigates the problem of outer synchronization between two complex networks with the same topological structure and timevarying coupling delay. The authors of [25] investigated the outer synchronization problem of complex networks with multiple coupling time-varying delays. However, in order to fit with the real world, complex networks with nondelayed and delayed coupling when the delay time is varying should be considered. To the best of our knowledge, there are few (if any) results concerning outer synchronization of complex networks with nondelayed and coupling time-varying delays until now. Therefore, how to solve the outer synchronization problem for complex networks with nondelayed and coupling time-varying delays still remains largely challenging.

Motivated by the above considerations, this paper aims to analyze the outer synchronization between two coupled complex networks with nondelayed and time-varying delays by two different control schemes. The main contribution in our work is as follows: firstly, the configuration matrices are not required to be assumed symmetric which means the networks can be either undirected or directed in the networks. Secondly, we deal with the situation of the networks with nondelayed and time-varying delayed couplings, which is more practical and can describe the actual applications better. Thirdly, based on Lyapunov stability theorem and linear matrix inequality (LMI), we investigate the networks and derive some criteria for the outer synchronization by using some pinning control scheme. Finally, we discuss the networks where the nondelayed coupling matrix does not need to be irreducible, and some sufficient conditions are obtained for achieving the outer synchronization by imposing the impulsive controllers.

The outline of this paper is organized as follows. In Section 2, we present the complex dynamical network model and introduce some necessary definitions, lemmas, and assumptions. In Section 3, we present the main theoretical analysis for outer synchronization by two methods. First, we discuss outer mixed synchronization through pinning control, and, then, we discuss outer synchronization via impulsive control. Numerical simulations to show the validity of the obtained theoretical results are also presented in Section 4. Finally, this paper is concluded in Section 5.

\section{Model Description and Preliminaries}

In this paper, we consider two coupled complex dynamical networks consisting of linearly coupled $N$ identical dynamical nodes, with each node being an $n$-dimensional dynamic system, respectively.

The drive coupled complex network is characterized by

$$
\begin{aligned}
\dot{x}_{i}(t)= & f\left(x_{i}(t)\right)+c_{1} \sum_{j=1}^{N} a_{i j} \Gamma_{1} x_{j}(t) \\
& +c_{2} \sum_{j=1}^{N} b_{i j} \Gamma_{2} x_{j}(t-\tau(t)), \quad i=1,2, \ldots, N .
\end{aligned}
$$

The response complex dynamical network with controllers is given as follows:

$$
\begin{aligned}
\dot{y}_{i}(t)= & f\left(y_{i}(t)\right)+c_{1} \sum_{j=1}^{N} a_{i j} \Gamma_{1} y_{j}(t) \\
& +c_{2} \sum_{j=1}^{N} b_{i j} \Gamma_{2} y_{j}(t-\tau(t))+u_{i}(t), \quad i=1,2, \ldots, N,
\end{aligned}
$$

where $x_{i}(t)=\left(x_{i}^{1}(t), x_{i}^{2}(t), \ldots, x_{i}^{n}(t)\right)^{T} \in R^{n}$ is the drive state variables of the $i$ th node and $y_{i}(t)=\left(y_{i}^{1}(t), y_{i}^{2}(t), \ldots\right.$, $\left.y_{i}^{n}(t)\right)^{T} \in R^{n}$ is the response state variables of the $i$ th node, $t \in[0,+\infty)$. The constants $c_{1}>0$ and $c_{2}>0$ denote the nondelayed and delayed coupling strength, respectively. The intrinsic function $f(\cdot): \mathbf{R}^{n} \rightarrow \mathbf{R}^{n}(i=1,2, \ldots, N)$ is continuous, which describes the local dynamics of nodes $i$, and $\Gamma_{1}=\operatorname{diag}\left(\gamma_{1}^{1}, \gamma_{1}^{2}, \ldots, \gamma_{1}^{n}\right)$ and $\Gamma_{2}=\operatorname{diag}\left(\gamma_{2}^{1}, \gamma_{2}^{2}, \ldots, \gamma_{2}^{n}\right)$ are positive definite diagonal inner coupling matrices. $A=$ $\left(a_{i j}\right) \in R^{N \times N}$ and $B=\left(b_{i j}\right) \in R^{N \times N}$ are the nondelayed and delayed weight configuration matrices, respectively. If there is a connection from the node $i$ to the node $j(j \neq i)$, $a_{i j} \neq 0$; otherwise, $a_{i j}=0$. So is the case with matrix $B$. Here, $A$ and $B$ are not required to be symmetric, which correspond to the direct network in the real world. $a_{i i}=$ $-\sum_{j=1, j \neq i}^{N} a_{i j}$, and $b_{i i}=-\sum_{j=1, j \neq i}^{N} b_{i j},(i=1,2, \ldots, N)$. The coupling time-varying delay $\tau(t)$ is a bounded and continuously differentiable function. Suppose that there exist positive constants $\alpha$ and $\tau$ satisfying $0 \leq \dot{\tau}(t) \leq \alpha<1$ and $0 \leq \tau(t) \leq \tau . u_{i}(t)(i=1,2, \ldots, N)$ is the linear controller for $i$ to design later.

Suppose $C\left([-\tau, 0], R^{n}\right)$ is the Banach space of continuous vector-valued functions mapping the interval $[-\tau, 0]$ into $R^{n}$ with the norm $\|\phi\|=\sup _{-\tau<s<0}\|\phi(s)\|$. For the functional differential equation (1), its initial conditions are given by $x_{i}(t)=\phi_{i}(t) \in C\left([-\tau, 0], R^{n}\right)$. It is assumed that (1) has a 
unique solution with respect to these initial conditions. For the functional differential equation (2), its initial conditions are given by $y_{i}(t)=\psi_{i}(t) \in C\left([-\tau, 0], R^{n}\right)$. And, at least, there exists a constant $i(i=1,2, \ldots, N)$ such that $\phi_{i}(t) \neq \psi_{i}(t)$ for $t \in[-\tau, 0] .\|\cdot\|$ refers to the Euclidean vector norm or the induced matrix 2-norm.

In the following, some preliminaries such as definitions, lemmas, and assumptions will be given, which would be used throughout the paper.

Definition 1. The drive networks (1) and the response networks (2) are said to be achieving complete outer synchronization, if, for any initial sates $\phi_{i}(t)$ and $\psi_{i}(t)$,

$$
\lim _{t \rightarrow \infty}\left\|y_{i}\left(t, \psi_{i}\right)-x_{i}\left(t, \phi_{i}\right)\right\|=0, \quad i=1,2, \ldots, N .
$$

Definition 2. Matrix $A=\left(a_{i j}\right) \in R^{N \times N}$ is said to belong to class $A 1$, denoted as $A \in A 1$, if

(1) $a_{i j} \geq 0, i \neq j, a_{i i}=-\sum_{j=1, j \neq i}^{N} a_{i j}, i=1,2, \ldots, N$,

(2) $A$ is irreducible.

If $A \in A 1$ is symmetrical, then one says that $A$ belongs to class $A 2$, denoted as $A \in A 2$.

Lemma 3 (see [25]). If matrix $A \in A 1$, then the following are valid.

(1) Real parts of the eigenvalues of $A$ are negative except an eigenvalue 0 with multiplicity 1.

(2) A has right eigenvalues $(1,1, \ldots, 1)^{T}$ corresponding to the eigenvalue 0 .

(3) Let $\xi=\left(\xi_{1}, \xi_{2}, \ldots, \xi_{N}\right)^{T}$ be the left eigenvector of $A$ corresponding to the eigenvalue 0 satisfying $\sum_{i=1}^{N} \xi_{i}=1$, and then one can let $\xi_{i}>0$ hold for all $i=1,2, \ldots, N$.

Lemma 4 (see [15]). The following linear matrix inequality (LMI):

$$
\left(\begin{array}{ll}
S_{11} & S_{12} \\
S_{12}^{T} & S_{22}
\end{array}\right)<0
$$

is equivalent to the following conditions:

$$
\begin{array}{ll}
\text { (1) } S_{11}<0, & S_{22}-S_{12}^{T} S_{11}^{-1} S_{12}<0 ; \\
\text { (2) } S_{22}<0, & S_{11}-S_{12} S_{22}^{-1} S_{12}^{T}<0,
\end{array}
$$

where $S_{11}$ and $S_{22}$ are symmetric matrices and $S_{12}$ is a matrix with suited dimensions.

Lemma 5. Let $u(t):\left[t_{0}-\tau, \infty\right) \rightarrow[0, \infty)$ satisfy the scalar impulsive differential inequality:

$$
\begin{gathered}
\dot{u}(t) \leq p u(t)+q u(t-\tau(t)), \quad t \neq t_{k}, \quad t \geq t_{0}, \\
u\left(t_{k}\right) \leq \alpha_{k} u\left(t_{k}^{-}\right), \quad t=t_{k}, \quad k=1,2, \ldots, \\
u(t)=\varphi(t), \quad t \in\left[t_{0}-\tau, t_{0}\right],
\end{gathered}
$$

where $p, q>0, \alpha_{k}>0, u(t)$ is continuous at $t \neq t_{k}, t \geq t_{0}$, $u\left(t_{k}\right)=u\left(t_{k}^{+}\right)=\lim _{t \rightarrow t_{k}^{+}} u(t), u\left(t_{k}^{-}\right)=\lim _{t \rightarrow t_{k}^{-}} u(t)$ exists, and $\varphi \in C\left(\left[t_{0}-\tau, t_{0}\right], R^{+}\right) . \tau(t)$ is a bounded and continuously differentiable function, and $0 \leq \dot{\tau}(t) \leq \alpha<1$ and $0 \leq \tau(t) \leq \tau$. Then

$$
u(t) \leq\left(\prod_{i=1}^{k} \alpha_{i}\right) e^{(p+q /(1-\alpha))\left(t-t_{0}+k \tau\right)}\left[\sup _{t_{0}-\tau \leq s \leq t_{0}} \varphi(s)\right]
$$

for $t \in\left[t_{k}, t_{k+1}\right)$

Proof. For $t \in\left[t_{k}, t_{k+1}\right)$, integrating both sides of inequality (6) from $t_{k}$ to $t$, we can obtain

$$
u(t)-u\left(t_{k}\right) \leq \int_{t_{k}}^{t}(p u(s)+q u(s-\tau(s))) d s .
$$

It is easy to get

$$
u(t) \leq u\left(t_{k}\right)+\int_{t_{k}-\tau}^{t}\left(p+\frac{q}{1-\alpha}\right) u(s) d s .
$$

We will prove the conclusion of Lemma 5 by induction. From inequality (6), when $t \in\left[t_{0}, t_{1}\right)$, we can get

$$
\begin{aligned}
\dot{u}(t) & \leq p u(t)+q \sup _{t-\tau \leq s \leq \tau} u(s) \\
& \leq p u(t)+\frac{q}{1-\alpha} \sup _{t-\tau \leq s \leq \tau} u(s) .
\end{aligned}
$$

By Lemma 3 in [26], for $t \in\left[t_{0}, t_{1}\right)$, we have

$$
u(t) \leq\left(\sup _{t_{0}-\tau \leq s \leq t_{0}} \varphi(s)\right) e^{(p+q /(1-\alpha))\left(t-t_{0}\right)} .
$$

This implies that the conclusion of Lemma 5 holds for $k=0$.

Under the inductive assumption that the conclusion (6) holds for some $k>0$, we will show that (6) still holds for $k+1$. For $t \in\left[t_{k+1}, t_{k+2}\right)$, without loss of generality, we assume that there are $l$ first class intermittent points, and then (9) can be rewritten as

$$
\begin{aligned}
u(t) \leq & u\left(t_{k+1}\right)+\int_{t_{k+1}-\tau}^{t_{k-l+1}}\left(p+\frac{q}{1-\alpha}\right) u(s) d s \\
& +\sum_{i=1}^{l} \int_{t_{k-i+1}}^{t_{k-i+2}}\left(p+\frac{q}{1-\alpha}\right) u(s) d s \\
& +\int_{t_{k+1}}^{t}\left(p+\frac{q}{1-\alpha}\right) u(s) d s .
\end{aligned}
$$

Using the same method as the proof of Lemma 1 in [13], we can get, for $t \in\left[t_{k+1}, t_{k+2}\right)$,

$$
u(t) \leq u\left(t_{k+1}\right) e^{(p+q /(1-\alpha))\left(t-t_{k+1}+\tau\right)}
$$

and by the inductive assumption and the second inequality of (6), we have

$$
\begin{aligned}
u(t) & \leq \alpha_{k+1} u\left(t_{k+1}^{-}\right) e^{(p+q /(1-\alpha))\left(t-t_{k+1}+\tau\right)} \\
& \leq\left(\prod_{i=1}^{k+1} \alpha_{i}\right)\left[\sup _{t_{0}-\tau \leq s \leq t_{0}} \varphi(s)\right] e^{(p+q /(1-\alpha))\left(t-t_{0}+(k+1) \tau\right)} .
\end{aligned}
$$


Hence, by induction, the conclusion of the lemma is attained for all $k \geq 0$.

Assumption 6. Suppose there exists a positive constant $L$ such that

$$
\|f(y)-f(x)\| \leq L\|y-x\|, \quad i=1,2, \ldots, N,
$$

for any $x, y \in R^{n}$, and the norm $\|x\|=\sqrt{x^{T} x}$.

Remark 7. In fact, there are many classical chaotic systems, such as Lorenz system, Chen system, Lü system, and Chua's circuit system, whose corresponding dynamical functions all satisfy the above assumption.

\section{Outer Synchronization Analysis}

3.1. Complete Outer Synchronization of Complex Networks by Pinning Control. In this subsection, we first investigate outer synchronization issue for two linear coupled complex networks with delay and nondelay by pinning control; here, the nondelay coupled matrix $A$ is not assumed to be symmetric, but it has to be irreducible. Then, we introduce some notation employed throughout this paper.

The error vector is as follows:

$$
\begin{gathered}
e_{i}(t)=y_{i}(t)-x_{i}(t), \\
e^{k}(t)=\left(e_{1}^{k}(t), e_{2}^{k}(t), \ldots, e_{N}^{k}(t)\right)^{T}, \\
e^{k}(t-\tau(t)) \\
=\left(e_{1}^{k}(t-\tau(t)), e_{2}^{k}(t-\tau(t)), \ldots, e_{N}^{k}(t-\tau(t))\right)^{T},
\end{gathered}
$$

and we design the negative feedback controllers corresponding to the response network (2) as

$$
u_{i}(t)= \begin{cases}-c_{1} d_{i} \Gamma_{1} e_{i}(t), & 1 \leq i \leq l<N \\ 0, & 1+l \leq i \leq N\end{cases}
$$

where $d_{i}>0, i=1,2, \ldots, l$, are the feedback control gains that can be suitably chosen. Without loss of generality, we can control the first $l(l<N)$ nodes.

Transforming the system yields the following error dynamical system:

$$
\begin{array}{r}
\dot{e}_{i}(t)=f\left(y_{i}(t)\right)-f\left(x_{i}(t)\right)+c_{1} \sum_{j=1}^{N} a_{i j} \Gamma_{1} e_{j}(t) \\
+c_{2} \sum_{j=1}^{N} b_{i j} \Gamma_{2} e_{j}(t-\tau(t))-c_{1} d_{i} \Gamma_{1} e_{i}(t), \\
i=1,2, \ldots, l,
\end{array}
$$

$$
\begin{gathered}
\dot{e}_{i}(t)=f\left(y_{i}(t)\right)-f\left(x_{i}(t)\right)+c_{1} \sum_{j=1}^{N} a_{i j} \Gamma_{1} e_{j}(t) \\
+c_{2} \sum_{j=1}^{N} b_{i j} \Gamma_{2} e_{j}(t-\tau(t)) \\
i=1+l, \ldots, N .
\end{gathered}
$$

Then we have the following results.

Theorem 8. Suppose that Assumption 6 holds and the drive network (1) with response network (2) can realize outer synchronization if the following condition is satisfied:

$$
L \Xi+\frac{1}{1-\alpha} I_{N}+c_{1} \gamma_{1}^{k}(\Xi \widetilde{A})^{s}+\frac{c_{2}^{2}\left(\gamma_{2}^{k}\right)^{2} \Xi B(\Xi B)^{T}}{4}<0
$$

for $k=1,2, \ldots, n$, where $\Xi=\operatorname{diag}\left\{\xi_{1}, \ldots, \xi_{N}\right\}, \widetilde{A}=A-D, D=$ $\operatorname{diag}\left\{d_{1}, \ldots, d_{l}, 0, \ldots, 0\right\} \in R^{N \times N}$, and $(\Xi \widetilde{A})^{s}=\left(\Xi \widetilde{A}+\widetilde{A}^{T} \Xi\right) / 2$.

Proof. Construct a Lyapunov-Krasovskii function in the form

$$
V(t)=\frac{1}{2} \sum_{i=1}^{N} \xi_{i} e_{i}^{T}(t) e_{i}(t)+\frac{1}{1-\alpha} \sum_{k=1}^{n} \int_{t-\tau(t)}^{t}\left(e^{k}(s)\right)^{T} e^{k}(s) d s .
$$

Then the derivative of $V(t)$ along the trajectories of (18) is

$$
\begin{aligned}
\dot{V}(t)= & \sum_{i=1}^{N} \xi_{i} e_{i}^{T}(t) \dot{e}_{i}(t)+\frac{1}{1-\alpha} \\
& \times \sum_{k=1}^{n}\left[\left(e^{k}(t)\right)^{T} e^{k}(t)-(1-\dot{\tau}(t))\right. \\
& \left.\times e^{k}(t-\tau(t))^{T} e^{k}(t-\tau(t))\right] \\
\leq & \sum_{i=1}^{N} \xi_{i} e_{i}^{T}(t)\left[\left(f\left(y_{i}(t)\right)-f\left(x_{i}(t)\right)\right)+c_{1} \sum_{j=1}^{N} a_{i j} \Gamma_{1} e_{j}(t)\right. \\
& -c_{1} \sum_{i=1}^{l} \xi_{i} e_{i}^{T}(t) d_{i} \Gamma_{1} e_{i}(t) \\
& +\sum_{k=1}^{n}\left[\frac{1}{1-\alpha}\left(e^{k} b_{i j} \Gamma_{2} e_{j}(t-\tau(t))\right]\right. \\
& -\left(e^{k}(t-\tau(t))\right)^{T} e^{k}(t)
\end{aligned}
$$


According to Assumption 6, one can obtain

$$
\begin{aligned}
\sum_{i=1}^{N} \xi_{i} e_{i}^{T}(t)\left(f\left(y_{i}(t)\right)-f\left(x_{i}(t)\right)\right) & \leq L \sum_{i=1}^{N} \xi_{i} e_{i}^{T}(t) e_{i}(t) \\
& =L \sum_{k=1}^{n}\left(e^{k}(t)\right)^{T} \Xi e^{k}(t) .
\end{aligned}
$$

Note that

$$
\begin{aligned}
c_{1} \sum_{i=1}^{N} \sum_{j=1}^{N} \xi_{i} e_{i}^{T}(t) a_{i j} \Gamma_{1} e_{j}(t) & =c_{1} \sum_{i=1}^{N} \sum_{j=1}^{N} a_{i j} \xi_{i}\left[\sum_{k=1}^{n} e_{i}^{k}(t) \gamma_{1}^{k} e_{j}^{k}(t)\right] \\
& =c_{1} \sum_{k=1}^{n} \gamma_{1}^{k}\left[\sum_{i=1}^{N} \sum_{j=1}^{N} a_{i j} \xi_{i} e_{i}^{k}(t) e_{j}^{k}(t)\right] \\
& =\sum_{k=1}^{n}\left(e^{k}(t)\right)^{T} c_{1} \gamma_{1}^{k}(\Xi A)^{s} e^{k}(t) .
\end{aligned}
$$

Then, it follows that

$$
\begin{gathered}
c_{1} \sum_{i=1}^{N} \sum_{j=1}^{N} \xi_{i} e_{i}^{T}(t) a_{i j} \Gamma e_{j}(t)-c_{1} \sum_{i=1}^{l} \xi_{i} d_{i} e_{i}^{T}(t) \Gamma_{1} e_{i}(t) \\
=\sum_{k=1}^{n}\left(e^{k}(t)\right)^{T} c_{1} \gamma_{1}^{k} \Xi(A-D) e^{k}(t) \\
=\sum_{k=1}^{n}\left(e^{k}(t)\right)^{T} c_{1} \gamma_{1}^{k}(\Xi \widetilde{A})^{s} e^{k}(t) .
\end{gathered}
$$

Using the same method as (23), we get

$$
\begin{aligned}
c_{2} \sum_{i=1}^{N} \sum_{j=1}^{N} \xi_{i} e_{i}^{T}(t) b_{i j} \Gamma e_{j}(t-\tau(t)) \\
=c_{2} \sum_{i=1}^{N} \sum_{j=1}^{N} b_{i j} \xi_{i}\left[\sum_{k=1}^{n} e_{i}^{k}(t) \gamma_{1}^{k} e_{j}^{k}(t-\tau(t))\right] \\
=\sum_{k=1}^{n}\left(e^{k}(t)\right)^{T} c_{2} \gamma_{2}^{k} \Xi B e^{k}(t-\tau(t)) .
\end{aligned}
$$

Substituting inequalities (22), (24), and (25) into (21), we obtain

$$
\begin{aligned}
& \dot{V}(t) \leq \sum_{k=1}^{n}[\left(e^{k}(t)\right)^{T}\left(L \Xi+\frac{1}{1-\alpha} I_{N}+c_{1} \gamma_{1}^{k}(\Xi \widetilde{A})^{s}\right) e^{k}(t) \\
&+\left(e^{k}(t)\right)^{T} c_{2} \gamma_{2}^{k} \Xi B e^{k}(t-\tau(t)) \\
&\left.-\left(e^{k}(t-\tau(t))\right)^{T} I_{N} e^{k}(t-\tau(t))\right] \\
&=\sum_{k=1}^{n}\left[\left(e^{k}(t)\right)^{T},\left(e^{k}(t-\tau(t))\right)^{T}\right] \Xi_{1}\left(\begin{array}{c}
e^{k}(t) \\
e^{k}(t-\tau(t))
\end{array}\right),
\end{aligned}
$$

where

$$
\Xi_{1}=\left(\begin{array}{cc}
L \Xi+\frac{1}{1-\alpha} I_{N}+c_{1} \gamma_{1}^{k} \Xi \widetilde{A} & \frac{c_{2} \gamma_{2}^{k} \Xi B}{2} \\
\frac{c_{2} \gamma_{2}^{k}(\Xi B)^{T}}{2} & -I_{N}
\end{array}\right),
$$

from Lemma 4 and the condition of Theorem 8 , is equating to $\Xi_{1}<0$. So we obtain $\dot{V}_{i}(t) \leq 0$. This implies

$$
\lim _{t \rightarrow \infty}\left\|e_{i}(t)\right\|=\lim _{t \rightarrow \infty}\left\|y_{i}(t)-x_{i}(t)\right\|=0, \quad i=1,2, \ldots, N .
$$

According to the Lyapunov stability theorem, the outer synchronization of network is achieved. This completes the proof.

Remark 9. In a large body of the existing literature, the coupling matrix is supposed to be symmetric, which is not practical. However, in this paper, the configuration matrix need not be symmetric. This means that the networks (1) and (2) are directed networks. The complex network structure in this paper is general and this theorem can be applied to a great many complex networks in the real world.

When the coupling delay is absent in the complex network, that is, $B=0$, the drive coupled complex network is characterized by

$$
\dot{x}_{i}(t)=f\left(x_{i}(t)\right)+c \sum_{j=1}^{N} a_{i j} \Gamma_{1} x_{j}(t), \quad i=1,2, \ldots, N,
$$

and the response coupled complex dynamical network is as follows:

$$
\dot{y}_{i}(t)=f\left(y_{i}(t)\right)+c \sum_{j=1}^{N} a_{i j} \Gamma_{1} y_{j}(t)+u_{i}(t), \quad i=1,2, \ldots, N
$$

where $u_{i}(t)$ is the same as (17).

Corollary 10. Under Assumption 6, if the condition

$$
L \Xi+c \gamma_{1}^{k}(\Xi \widetilde{A})^{s}<0
$$

holds, then the drive systems (29) with response system (30) can achieve outer synchronization.

For the proof of Corollary 10, we choose the Lyapunov function as

$$
V(t)=\sum_{i=1}^{N} \xi_{i} e_{i}^{T}(t) e_{i}(t)
$$

Then, we can follow the proof of Theorem 8 to get the results above. We omitted details here.

Remark 11. The results in Corollary 10 correspond to Theorem 1 in [23] when $H=I$, which means the problem 
investigated in [23] reaches complete outer synchronization. The results we obtained here are more general since the dynamical behaviors in our complex networks (29) and (30) are comprehensive. In some sense, the results in [23] could be seen as a special case of our results.

When the nondelay coupled matrix $A$ is symmetric and irreducible, the left eigenvector of $A$ corresponding to eigenvalue 0 is $\xi=(1 / N, 1 / N, \ldots, 1 / N)$, and we could have the following results.

Corollary 12. Under Assumption 6, if A is symmetric and irreducible and the condition

$$
\left(L+\frac{1}{1-\alpha}\right) I_{N}+c_{1} \gamma_{1}^{k} \widetilde{A}+\frac{c_{2}^{2}\left(\gamma_{2}^{k}\right)^{2} B B^{T}}{4}<0
$$

holds, then the networks achieve outer synchronization.

3.2. Outer Synchronization of Complex Networks by Impulsive Control. In this section, we discuss outer synchronization for two linear coupled complex networks with delay and nondelay by impulsive control. It should be mentioned that the coupling matrix $A$ is irreducible in Theorem 8 when we use pinning control to get outer synchronization in the subsection above. However, in this subsection, the nondelay coupled matrix $A$ is not necessarily assumed to be symmetric and irreducible when we use impulsive control strategy.

For achieving outer synchronization of two networks, the impulsive controllers can be designed as follows:

$$
u_{i}(t)=\sum_{k=1}^{\infty} B_{i k}\left(y_{i}\left(t^{-}\right)-x_{i}\left(t^{-}\right)\right) \delta\left(t-t_{k}\right),
$$

where the impulsive instant sequence $\left\{t_{k}\right\}_{k=1}^{+\infty}$ satisfies $t_{k-1}<$ $t_{k}$ and $\lim _{k \rightarrow \infty} t_{k}=+\infty$. Matrix $B_{i k} \in R^{n \times n}$ is the states impulses gain matrix at the moment $t_{k}$. And $\delta(\cdot)$ is the Dirac impulsive function; that is,

$$
\delta\left(t-t_{k}\right)= \begin{cases}1, & t=t_{k} \\ 0, & t \neq t_{k}\end{cases}
$$

The response network with impulsive control can be expressed as follows:

$$
\begin{gathered}
\dot{y}_{i}(t)=f\left(y_{i}(t)\right)+c_{1} \sum_{j=1}^{N} a_{i j} \Gamma_{1} y_{j}(t) \\
\quad+c_{2} \sum_{j=1}^{N} b_{i j} \Gamma_{2} y_{j}(t-\tau(t)), \quad t \neq t_{k}, \quad t \geq t_{0}, \\
\Delta y_{i}=y_{i}\left(t_{k}^{+}\right)-y_{i}\left(t_{k}^{-}\right) \\
=B_{i k}\left(y_{i}\left(t_{k}^{-}\right)-x_{i}\left(t_{k}^{-}\right)\right), \quad t=t_{k}, k=1,2, \ldots, \\
\quad y_{i}(t)=\psi_{i}(t), \quad t \in[-\tau, 0],
\end{gathered}
$$

where $y_{i}\left(t_{k}^{+}\right)=\lim _{t \rightarrow t_{k}^{+}} y_{i}(t)$ and $y_{i}\left(t_{k}^{-}\right)=\lim _{t \rightarrow t_{k}^{-}} y_{i}(t)$. Here, we suppose that $y_{i}\left(t_{k}\right)=y_{i}\left(t_{k}^{+}\right)$, which means that the solution $y(t)$ of (36) is right-hand continuous at the impulsive moment $t_{k}$.

Let $e_{i}(t)=y_{i}(t)-x_{i}(t)$, and then the error system can be written as follows:

$$
\begin{gathered}
\dot{e}_{i}(t)=f\left(y_{i}(t)\right)-f\left(x_{i}(t)\right)+c_{1} \sum_{j=1}^{N} a_{i j} \Gamma_{1} e_{j}(t) \\
+c_{2} \sum_{j=1}^{N} b_{i j} \Gamma_{2} e_{j}(t-\tau(t)), \quad t \neq t_{k}, \quad t \geq t_{0}, \\
\Delta e_{i}=e_{i}\left(t_{k}^{+}\right)-e_{i}\left(t_{k}^{-}\right)=B_{i k} e_{i}\left(t_{k}^{-}\right), \quad t=t_{k}, k=1,2, \ldots, \\
e_{i}(t)=\psi_{i}(t)-\phi_{i}(t), \quad t \in[-\tau, 0],
\end{gathered}
$$

where the goal in this section is to set the control gains matrix $B_{i k}$ and the impulsive distance $\Delta t_{k}=t_{k}-t_{k-1},(k=1,2, \ldots)$ such that the error $\left\|e_{i}(t)\right\|$ converges to 0 , which implies the impulsive control synchronization of (37) is completed for arbitrary initial conditions.

Theorem 13. If Assumption 6 is satisfied, the drive system (1) and the response system (2) can achieve outer synchronization under the impulsive controller (34) if there exists a positive constant $\eta$, such that

$$
\left(\beta+\frac{c_{2}}{1-\alpha}\right)\left(1+\frac{\tau}{T_{\min }}\right)+\frac{2 \ln \left|\sigma_{k}\right|}{T_{\max }}<-\eta
$$

holds, where

$$
\beta=2 L+\lambda_{m}\left(2 c_{1}\left(A \otimes \Gamma_{1}\right)^{s}+c_{2}\left(B \otimes \Gamma_{2}\right)\left(B \otimes \Gamma_{2}\right)^{T}\right),
$$

and $\sigma_{k}=\max _{1 \leq i \leq N}\left\|I_{n}+B_{i k}\right\|, k \in Z^{+}, T_{\min }=\min \left\{t_{k}-t_{k-1} \mid\right.$ $\left.k \in Z^{+}\right\}, T_{\max }=\max \left\{t_{k}-t_{k-1} \mid k \in Z^{+}\right\}$, and $\lambda_{m}(\cdot)$ is the largest eigenvalue of a matrix.

Proof. Let $e(t)=\left(e_{1}^{T}(t), e_{2}^{T}(t), \ldots, e_{N}^{T}(t)\right)^{T}$. Choose the following Lyapunov function:

$$
V(t)=e^{T}(t) e(t) .
$$

For $t \neq t_{k}, k \in N$, taking the time derivative of the Lyapunov function (40) along the trajectories of (37), we have

$$
\begin{gathered}
\dot{V}(t)=2 \sum_{i=1}^{N} e_{i}^{T}(t) \dot{e}_{i}(t) \\
=2 \sum_{i=1}^{N}\left[e_{i}^{T}(t)\left(f\left(y_{i}(t)\right)-f\left(x_{i}(t)\right)\right)\right. \\
+c_{1} \sum_{j=1}^{N} a_{i j} e_{i}^{T}(t) \Gamma_{1} e_{j}(t) \\
\left.+c_{2} \sum_{j=1}^{N} b_{i j} e_{i}^{T}(t) \Gamma_{2} e_{j}(t-\tau(t))\right] .
\end{gathered}
$$


From Assumption 6, one can obtain

$$
\begin{aligned}
2 \sum_{i=1}^{N} e_{i}^{T}(t)\left(f\left(y_{i}(t)\right)-f\left(x_{i}(t)\right)\right) & \leq 2 \sum_{i=1}^{N} L e_{i}^{T}(t) e_{i}(t) \\
& =2 L V(t) .
\end{aligned}
$$

Notice that

$$
\begin{aligned}
2 c_{1} \sum_{i=1}^{N} \sum_{j=1}^{N} e_{i}^{T}(t) a_{i j} \Gamma_{1} e_{j}(t) & =2 c_{1} e^{T}(t)\left(A \otimes \Gamma_{1}\right) e(t) \\
& \leq 2 c_{1} e^{T}(t)\left(A \otimes \Gamma_{1}\right)^{s} e(t) .
\end{aligned}
$$

The third term in (41) can be rewritten as

$$
\begin{aligned}
2 c_{2} \sum_{i=1}^{N} \sum_{j=1}^{N} b_{i j} e_{i}^{T}(t) \Gamma_{2} e_{j}(t-\tau(t)) \\
=2 c_{2} e^{T}(t)\left(B \otimes \Gamma_{2}\right) e(t-\tau(t)) \\
\leq c_{2}\left[e^{T}(t)\left(B \otimes \Gamma_{2}\right)\left(B \otimes \Gamma_{2}\right)^{T} e(t)\right. \\
\left.\quad+e^{T}(t-\tau(t)) e(t-\tau(t))\right] .
\end{aligned}
$$

Combining (43) and (44), we have

$$
\begin{aligned}
2 \sum_{i=1}^{N} \sum_{j=1}^{N}\left[c_{1} e_{i}^{T}(t) a_{i j} \Gamma_{1} e_{j}(t)+b_{i j} e_{i}^{T}(t) \Gamma_{2} e_{j}(t-\tau(t))\right] \\
=e^{T}(t)\left(\left(2 c_{1} A \otimes \Gamma_{1}\right)+c_{2}\left(B \otimes \Gamma_{2}\right)\left(B \otimes \Gamma_{2}\right)^{T}\right) e(t) \\
\quad+c_{2} V(t-\tau(t)) \\
\leq \lambda_{m}\left(2 c_{1}\left(A \otimes \Gamma_{1}\right)^{s}+c_{2}\left(B \otimes \Gamma_{2}\right)\left(B \otimes \Gamma_{2}\right)^{T}\right) V(t) \\
\quad+c_{2} V(t-\tau(t)) .
\end{aligned}
$$

Referring to the inequalities (42) and (45), for $t \neq t_{k}, k \in Z^{+}$, it follows from (41) that

$$
\begin{aligned}
\dot{V}(t) \leq & {[2 L} \\
& \left.+\lambda_{m}\left(2 c_{1}\left(A \otimes \Gamma_{1}\right)^{s}+c_{2}\left(B \otimes \Gamma_{2}\right)\left(B \otimes \Gamma_{2}\right)^{T}\right)\right] V(t) \\
& +c_{2} V(t-\tau(t)) \\
= & \beta V(t)+c_{2} V(t-\tau(t)),
\end{aligned}
$$

where

$$
\beta=2 L+\lambda_{m}\left(2 c_{1}\left(A \otimes \Gamma_{1}\right)^{s}+c_{2}\left(B \otimes \Gamma_{2}\right)\left(B \otimes \Gamma_{2}\right)^{T}\right) .
$$

When $t=t_{k}, k \in Z^{+}$, one has

$$
\begin{aligned}
V\left(t_{k}\right) & =\sum_{i=1}^{N} e_{i}^{T}\left(t_{k}^{+}\right) e_{i}\left(t_{k}^{+}\right) \\
& =\sum_{i=1}^{N} e_{i}^{T}\left(t_{k}^{-}\right)\left(I_{n}+B_{i k}\right)^{T}\left(I_{n}+B_{i k}\right) e_{i}\left(t_{k}^{-}\right)
\end{aligned}
$$

$$
\begin{aligned}
& \leq \max _{1 \leq i \leq N}\left\|I_{n}+B_{i k}\right\|^{2} \sum_{i=1}^{N} e_{i}^{T}\left(t_{k}^{-}\right) e_{i}\left(t_{k}^{-}\right) \\
& =\sigma_{k}^{2} V\left(t_{k}^{-}\right),
\end{aligned}
$$

where $\sigma_{k}=\max _{1 \leq i \leq N}\left\|I_{n}+B_{i k}\right\|$.

Thus, employing Lemma 5, from (45) and (46), for $t \in$ $\left[t_{k}, t_{k+1}\right)$, we have

$$
V(t) \leq\left[\sup _{t_{0}-\tau \leq s \leq t_{0}} V(s)\right]\left(\prod_{i=1}^{k} \sigma_{k}^{2}\right) e^{\left(\beta+c_{2} /(1-\alpha)\right)\left(t-t_{0}+k \tau\right)} .
$$

Let $T_{\min }=\min \left\{t_{k}-t_{k-1} \mid k \in Z^{+}\right\}$and $T_{\max }=\max \left\{t_{k}-t_{k-1} \mid\right.$ $\left.k \in Z^{+}\right\}$, and then

$$
\begin{aligned}
V(t) & \leq\left[\sup _{t_{0}-\tau \leq s \leq t_{0}} V(s)\right] e^{\left(\beta+c_{2} /(1-\alpha)\right)\left(t-t_{0}+k \tau\right)+2 k \ln \left|\sigma_{k}\right|} \\
& \leq\left[\sup _{t_{0}-\tau \leq s \leq t_{0}} V(s)\right] e^{\left[\left(\beta+c_{2} /(1-\alpha)\right)\left(1+\tau / T_{\min }\right)+2\left(\ln \left|\sigma_{k}\right| / T_{\max }\right)\right]\left(t-t_{0}\right)} .
\end{aligned}
$$

From condition (38) of Theorem 13, we get

$$
V(t) \leq\left[\sup _{t_{0}-\tau \leq s \leq t_{0}} V(s)\right] e^{-\eta\left(t-t_{0}\right)} .
$$

Thus, we can obtain that

$$
\left\|e_{i}(t)\right\| \leq\left[\sup _{t_{0}-\tau \leq s \leq t_{0}} V(s)\right]^{1 / 2} e^{-(\eta / 2)\left(t-t_{0}\right)}, \quad i=1,2, \ldots, N
$$

This means the impulsive outer synchronization between complex network (1) and network (2) is realized. Thus, we complete the proof work.

When the nondelayed coupling matrix $A=0$,

$$
\begin{array}{r}
\dot{x}_{i}(t)=f\left(x_{i}(t)\right)+c \sum_{j=1}^{N} b_{i j} \Gamma_{2} y_{j}(t-\tau(t)), \\
i=1,2, \ldots, N, \\
\dot{y}_{i}(t)=f\left(y_{i}(t)\right)+c \sum_{j=1}^{N} b_{i j} \Gamma_{2} y_{j}(t-\tau(t)), \\
t \neq t_{k}, t \geq t_{0},
\end{array}
$$

$$
\begin{gathered}
\Delta y_{i}=y_{i}\left(t_{k}^{+}\right)-y_{i}\left(t_{k}^{-}\right)=B_{i k}\left(y_{i}\left(t_{k}^{-}\right)-x_{i}\left(t_{k}^{-}\right)\right), \\
t=t_{k}, k=1,2, \ldots, \\
y_{i}(t)=\psi_{i}(t), \quad t \in[-\tau, 0] .
\end{gathered}
$$


Corollary 14. Suppose Assumption 6 is satisfied, and the network (53) can achieve outer synchronization if there exists a positive constant $\eta$, such that

$$
\left(\beta+\frac{c}{1-\alpha}\right)\left(1+\frac{\tau}{T_{\min }}\right)+\frac{2 \ln \left|\sigma_{k}\right|}{T_{\max }}<-\eta
$$

holds, where

$$
\beta=2 L+\lambda_{m}\left(c\left(B \otimes \Gamma_{2}\right)\left(B \otimes \Gamma_{2}\right)^{T}\right),
$$

and $\sigma_{k}=\max _{1 \leq i \leq N}\left\|I_{n}+B_{i k}\right\|, T_{\min }=\min \left\{t_{k}-t_{k-1} \mid k \in Z^{+}\right\}$, $T_{\text {max }}=\max \left\{t_{k}-t_{k-1} \mid k \in Z^{+}\right\}$, and $\lambda_{m}(\cdot)$ stands for the largest eigenvalue of a matrix.

Remark 15. Mentions should be made on Theorem 2 in [23]. In fact, (13) in [23] corresponds to (53) in this paper if we set $H=I_{n}$ in [23]. Both negative feedback controllers and impulsive controllers are used in [23] to make the complex network (53) to achieve outer synchronization. Compared with [23], only impulsive controllers are made use of. As a result, the criteria we presented in this paper are easier than those in [23].

\section{Numerical Simulations}

In this section, we give numerical simulation to verify and demonstrate the effectiveness of the proposed method. In order to verify our results, we consider the driving complex network (1) as

$$
\begin{aligned}
\dot{x}_{i}(t)= & f\left(x_{i}(t)\right)+c_{1} \sum_{j=1}^{N} a_{i j} \Gamma_{1} x_{j}(t) \\
& +c_{2} \sum_{j=1}^{N} b_{i j} \Gamma_{2} x_{j}(t-\tau(t)), \quad i=1,2,3,4 .
\end{aligned}
$$

Example 16. We take the Lorenz system as the local node dynamics, which is shown in Figure 1. Its dynamical equations in a dimensionless form are as follows:

$$
\begin{gathered}
\dot{x}_{1}=10\left(x_{2}-x_{1}\right), \\
\dot{x}_{2}=28 x_{1}-x_{2}-x_{1} x_{3}, \\
\dot{x}_{3}=x_{1} x_{2}-\frac{8}{3} x_{3},
\end{gathered}
$$

where $\left(x_{1}, x_{2}, x_{3}\right)^{T} \in R^{3}$ is the state variables group of unified chaotic system. The initial condition is randomly chosen. We choose $\tau(t)=0.01-0.01 e^{-t}, \alpha=0.01, c_{1}=40, c_{2}=0.01$, $\Gamma_{1}=\operatorname{diag}\{5,5,5\}$, and $\Gamma_{2}=\operatorname{diag}\{0.2,0.2,0.2\}$. Similar to the verification in [23] with $L=150$, clearly, Assumption 6 is verified. Choosing the asymmetric coupling configuration matrices

$$
\begin{aligned}
A & =\left(\begin{array}{cccc}
-2 & 1 & 0 & 1 \\
1 & -3 & 2 & 0 \\
0 & 3 & -4 & 1 \\
2 & 0 & 0 & -2
\end{array}\right), \\
B & =\left(\begin{array}{cccc}
-2 & 1 & 1 & 0 \\
0 & -2 & 1 & 1 \\
1 & 0 & -2 & 1 \\
1 & 1 & 0 & -2
\end{array}\right)
\end{aligned}
$$

by calculation, the left eigenvector of $A$ corresponding to the eigenvalue 0 is $\xi=(3 / 8,2 / 8,1 / 8,2 / 8)^{T}$. We control the second node of the system with coupling strength 52 and the third node 10 with coupling strength 48 by pinning control, and then all the conditions in Theorem 8 are satisfied, so the asymmetric coupled network (21) can achieve outer synchronization. The simulation results are given in Figure 2, and we can observe that outer synchronization state is realized.

Example 17. Now, let us consider the outer exponential synchronization of complex networks. Again, the network model is the same as Example 16. Hence $\lambda_{m}\left(2 c_{1}\left(A \otimes \Gamma_{1}\right)^{s}+\right.$ $\left.c_{2}\left(B \otimes \Gamma_{2}\right)\left(B \otimes \Gamma_{2}\right)^{T}\right)=6.2435, T_{\max }=T_{\min }=0.01$, let

$$
B_{i k}=\left(\begin{array}{ccc}
-1.04 & 0 & 0 \\
0 & -1.04 & 0 \\
0 & 0 & -1.04
\end{array}\right) \text {, }
$$

and then $\sigma=0.04$. Then all the conditions in Theorem 13 are satisfied, and $\eta=31.0662$, so the asymmetric coupled network (21) can achieve outer synchronization. The simulation results are given in Figure 3. It can be seen clearly from Figure 3 that outer synchronization state is realized.

\section{Conclusion}

In this paper, we considered the outer synchronization between two complex networks with time nondelayed and time-varying delayed couplings under different control laws. First of all, we handled the outer synchronization by pinning control and derived some sufficient criteria based on Lyapunov stability theorem and LMI. Particularly, we obtain two corollaries, which are single nondelay coupled networks cases, and the coupling matrixes $A$ and $B$ are symmetric and irreducible. On the other side, we discuss the outer synchronization between two networks by impulsive control. Some sufficient conditions are derived by imposing the impulsive controllers to the nodes. In addition, we also present some corollaries. What is more, numerical simulations for two coupled complex networks which are composed of unified chaotic systems are given to demonstrate the effectiveness and feasibility of the schemes. 


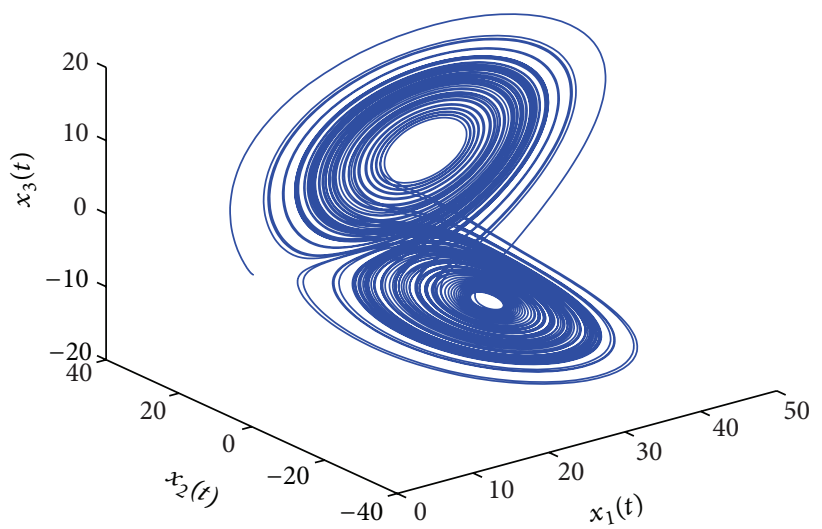

FIgURE 1: The attractor of the Lorenz system.

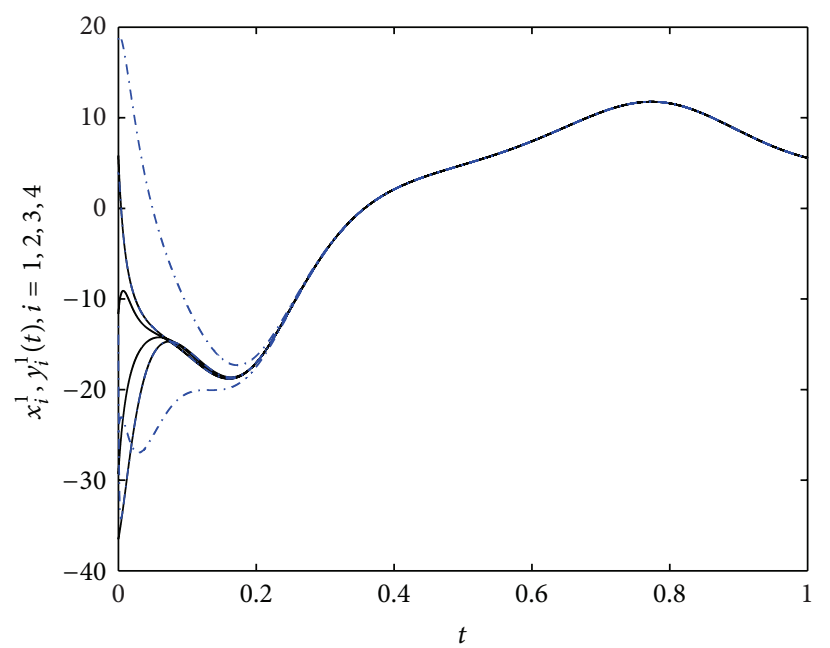

(a)

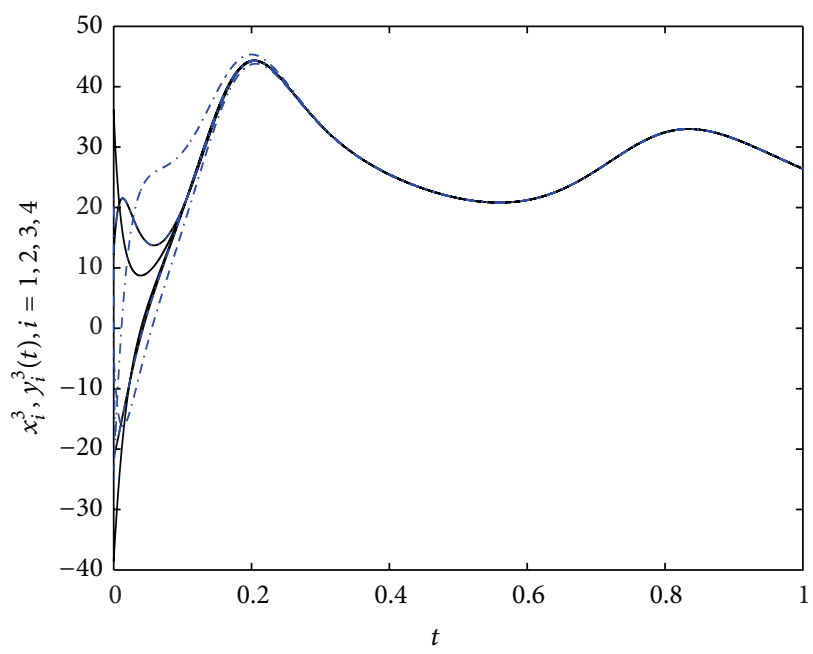

(c)

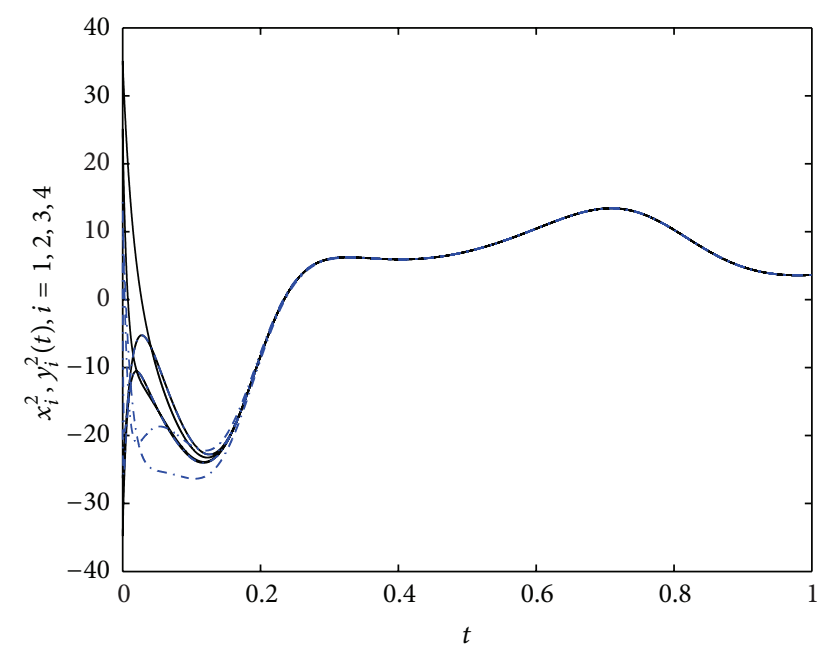

(b)

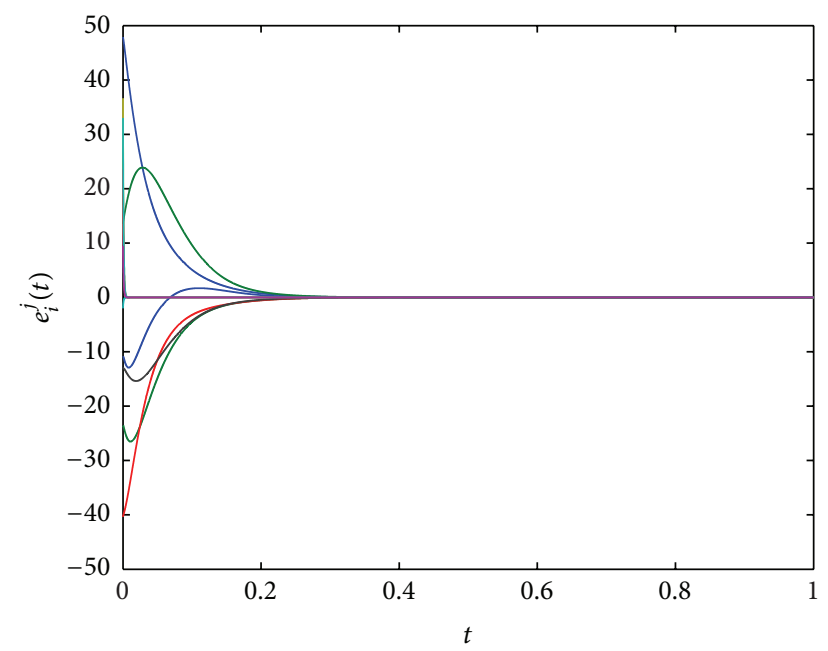

(d)

FIGURE 2: Time evolution between node $x_{i}^{k}$ and node $y_{i}^{k}, i=1,2,3,4, k=1,2,3$, and error evolution between drive and response networks under pinning control. 


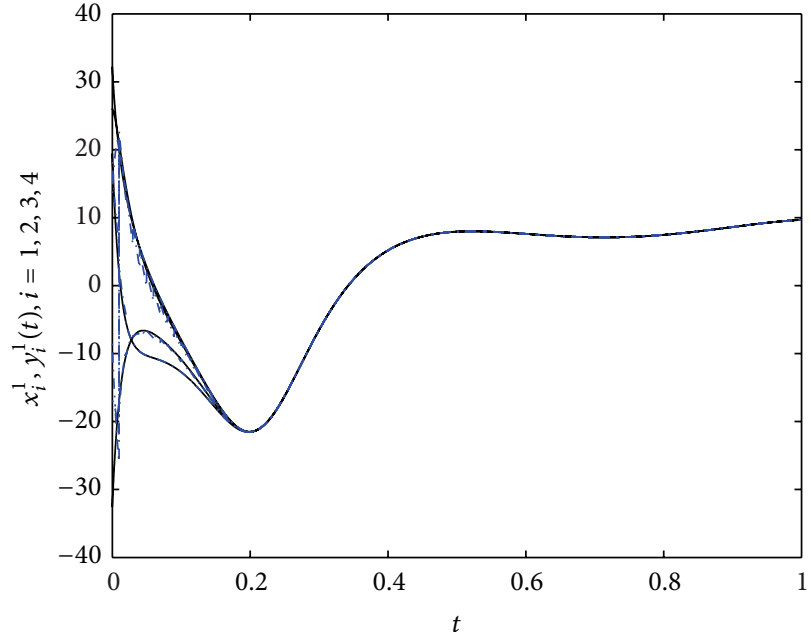

(a)

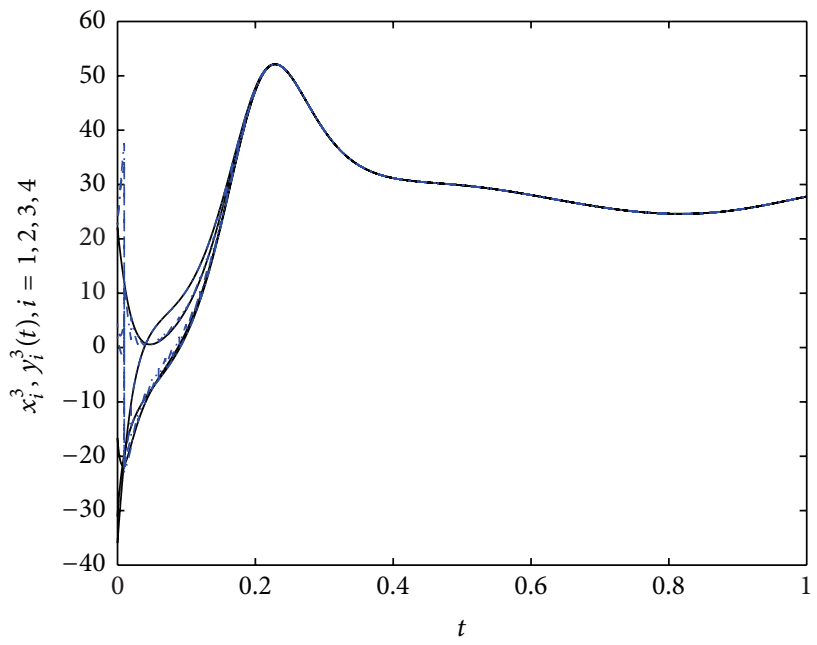

(c)

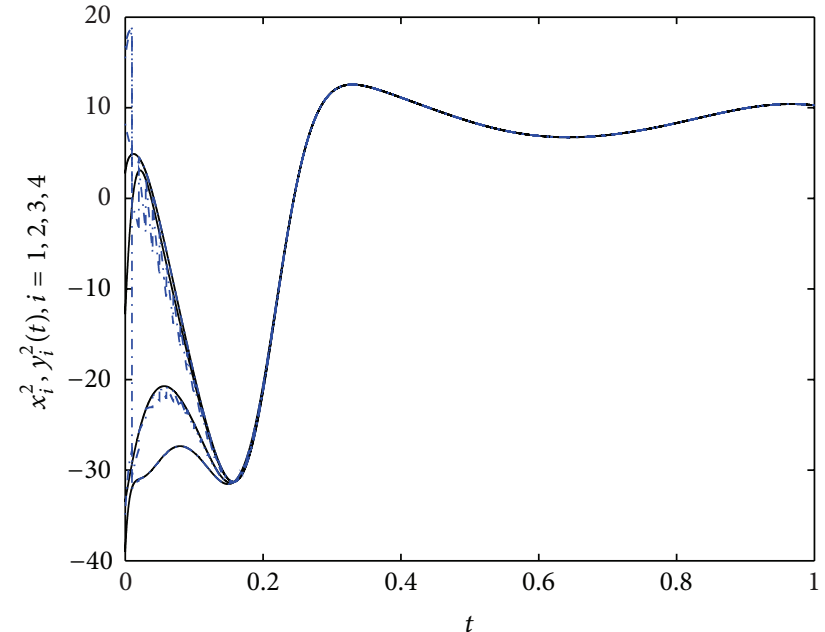

(b)

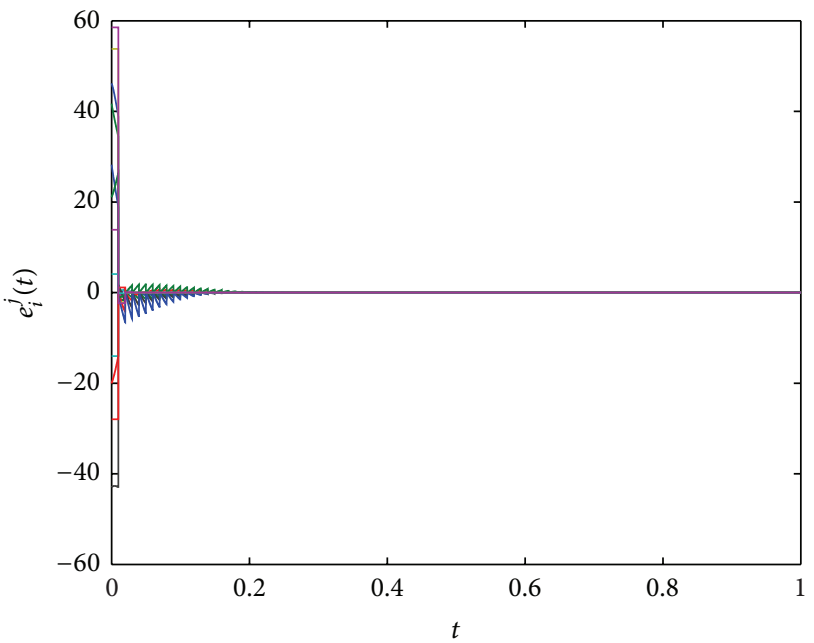

(d)

Figure 3: Time evolution between node $x_{i}^{k}$ and node $y_{i}^{k}, i=1,2,3,4, k=1,2,3$, and error evolution between drive and response networks under impulsive control.

\section{Conflict of Interests}

The authors declare that there is no conflict of interests regarding the publication of this paper.

\section{Acknowledgments}

The authors thank the referees and the editor for their valuable comments on this paper. This work is supported by the National Natural Science Foundation of China (Grants nos. 61273220 and 61373087) and the Shenzhen Basic Research Project (JCYJ20120613105730482).

\section{References}

[1] A. Arenas, A. Guilera, J. Kurths, Y. Moreno, and C. Zhou, "Synchronization in complex networks," Physics Reports, vol. 469, no. 3, pp. 93-153, 2008.
[2] D. C. Yu, M. Righero, and L. Kocarev, "Estimating topology of networks," Physical Review Letters, vol. 97, no. 18, Article ID 188701, 2006.

[3] S. H. Strogatz and I. Stewart, "Coupled oscillators and biological synchronization," Scientific American, vol. 269, no. 6, pp. 102109, 1993.

[4] C. M. Gray, "Synchronous oscillations in neuronal systems: mechanisms and functions," Journal of Computational Neuroscience, vol. 1, no. 1-2, pp. 11-38, 1994.

[5] M. D. Vieira, "Chaos and synchronized chaos in an earthquake model," Physical Review Letters, vol. 82, no. 1, pp. 201-204, 1999.

[6] L. Kuhnert, K. I. Agladze, and V. I. Krinsky, "Image processing using light-sensitive chemical waves," Nature, vol. 337, no. 6204, pp. 244-247, 1989.

[7] S. H. Wang, J. Y. Kuang, J. H. Li, Y. L. Luo, H. P. Lu, and G. Hu, "Chaos-based secure communications in a large community," Physical Review E, vol. 66, no. 6, Article ID 065202, 2002. 
[8] Y. Wu, "Adaptive impulsive outer synchronization between drive-response dynamical networks," Communications in Theoretical Physics, vol. 61, no. 50, pp. 590-594, 2014.

[9] X. F. Wang and G. Chen, "Pinning control of scale-free dynamical networks," Physica A, vol. 310, no. 3-4, pp. 521-531, 2002.

[10] T. Chen, X. Liu, and W. Lu, "Pinning complex networks by a single controller," IEEE Transactions on Circuits and Systems I: Regular Papers, vol. 54, no. 6, pp. 1317-1326, 2007.

[11] L. Xiang and J. J. H. Zhu, "On pinning synchronization of general coupled networks," Nonlinear Dynamics, vol. 64 , no. 4 , pp. 339-348, 2011.

[12] W. Xia and J. Cao, "Pinning synchronization of delayed dynamical networks via periodically intermittent control," Chaos, vol. 19, no. 1, Article ID 013120, 8 pages, 2009.

[13] J. Zhou, Q. Wu, and L. Xiang, "Impulsive pinning complex dynamical networks and applications to firing neuronal synchronization," Nonlinear Dynamics, vol. 69, no. 3, pp. 1393-1403, 2012.

[14] E. Montbrió, J. Kurths, and B. Blasius, "Synchronization of two interacting populations of oscillators," Physical Review E, vol. 70, Article ID 056125, 2004.

[15] J. Lu, D. W. C. Ho, J. Cao, and J. Kurths, "Single impulsive controller for globally exponential synchronization of dynamical networks," Nonlinear Analysis: Real World Applications, vol. 14, no. 1, pp. 581-593, 2013.

[16] J. D. Cao, D. W. C. Ho, and Y. Q. Yang, "Projective synchronization of a class of delayed chaotic systems via impulsive control," Physics Letters A, vol. 373, no. 35, pp. 3128-3133, 2009.

[17] B. Liu, X. Liu, G. Chen, and H. Wang, "Robust impulsive synchronization of uncertain dynamical networks," IEEE Transactions on Circuits and Systems. I: Regular Papers, vol. 52, no. 7, pp. 1431-1441, 2005.

[18] C. Li, W. Sun, and J. Kurths, "Synchronization between two coupled complex networks," Physical Review E, vol. 76, no. 4, Article ID 046204, 2007.

[19] Z. Li and X. Xue, "Outer synchronization of coupled networks using arbitrary coupling strength," Chaos, vol. 20, no. 2, Article ID 023106, 7 pages, 2010.

[20] W. Sun, Z. Z. Yan, S. H. Chen, and J. H. Lü, “Outer synchronization of complex networks by impulse," Communications in Theoretical Physics, vol. 56, no. 5, pp. 885-890, 2011.

[21] J. W. Wang, Q. H. Ma, Z. Li, and S. A. E. Mohammed, "Mixed outer synchronization of coupled complex networks with timevarying coupling delay," Chaos, vol. 21, Article ID 013121, pp. 1-8, 2011.

[22] S. Zheng, "Inner-outer synchronization analysis of two complex networks with delayed and non-delayed coupling," Journal of Information and Computing Science, vol. 7, no. 1, pp. 11-18, 2012.

[23] P. He, C. G. Jing, T. Fan, and C. Z. Chen, "Outer synchronization of complex networks with multiple coupling time-varying delays," International Journal of Control and Automation, vol. 6, no. 4, pp. 197-216, 2013.

[24] S. Zheng and W. Shao, "Mixed outer synchronization of dynamical networks with nonidentical nodes and output coupling," Nonlinear Dynamics, vol. 73, no. 4, pp. 2343-2352, 2013.

[25] X. Liu and T. Chen, "Synchronization analysis for nonlinearlycoupled complex networks with an asymmetrical coupling matrix," Physica A, vol. 387, no. 16-17, pp. 4429-4439, 2008.

[26] W. Xia and J. Cao, "Pinning synchronization of delayed dynamical networks via periodically intermittent control," Chaos, vol. 19, Article ID 013120, 2009. 


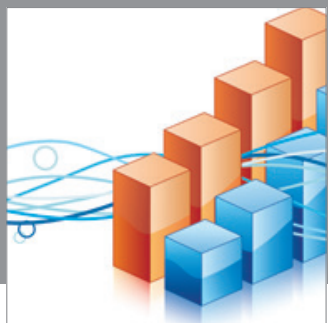

Advances in

Operations Research

mansans

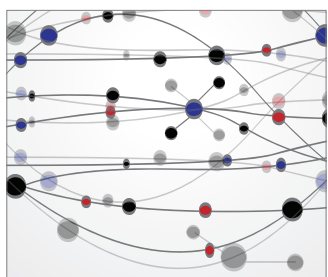

The Scientific World Journal
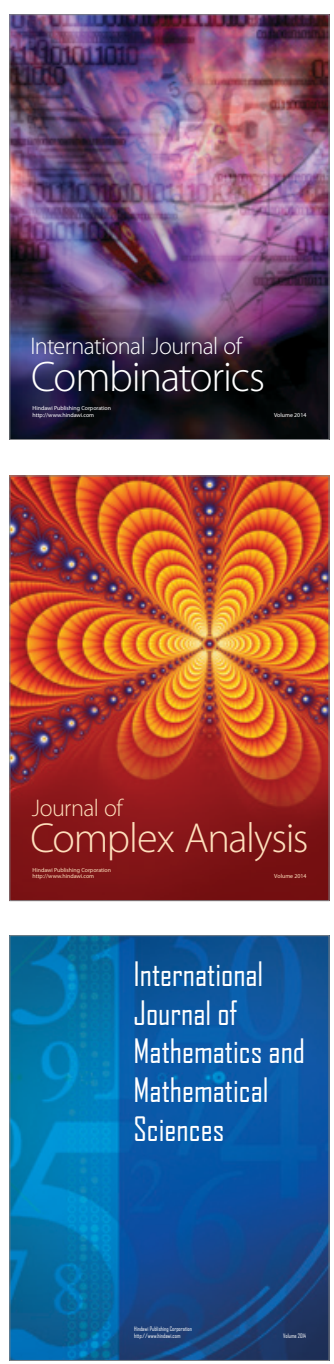
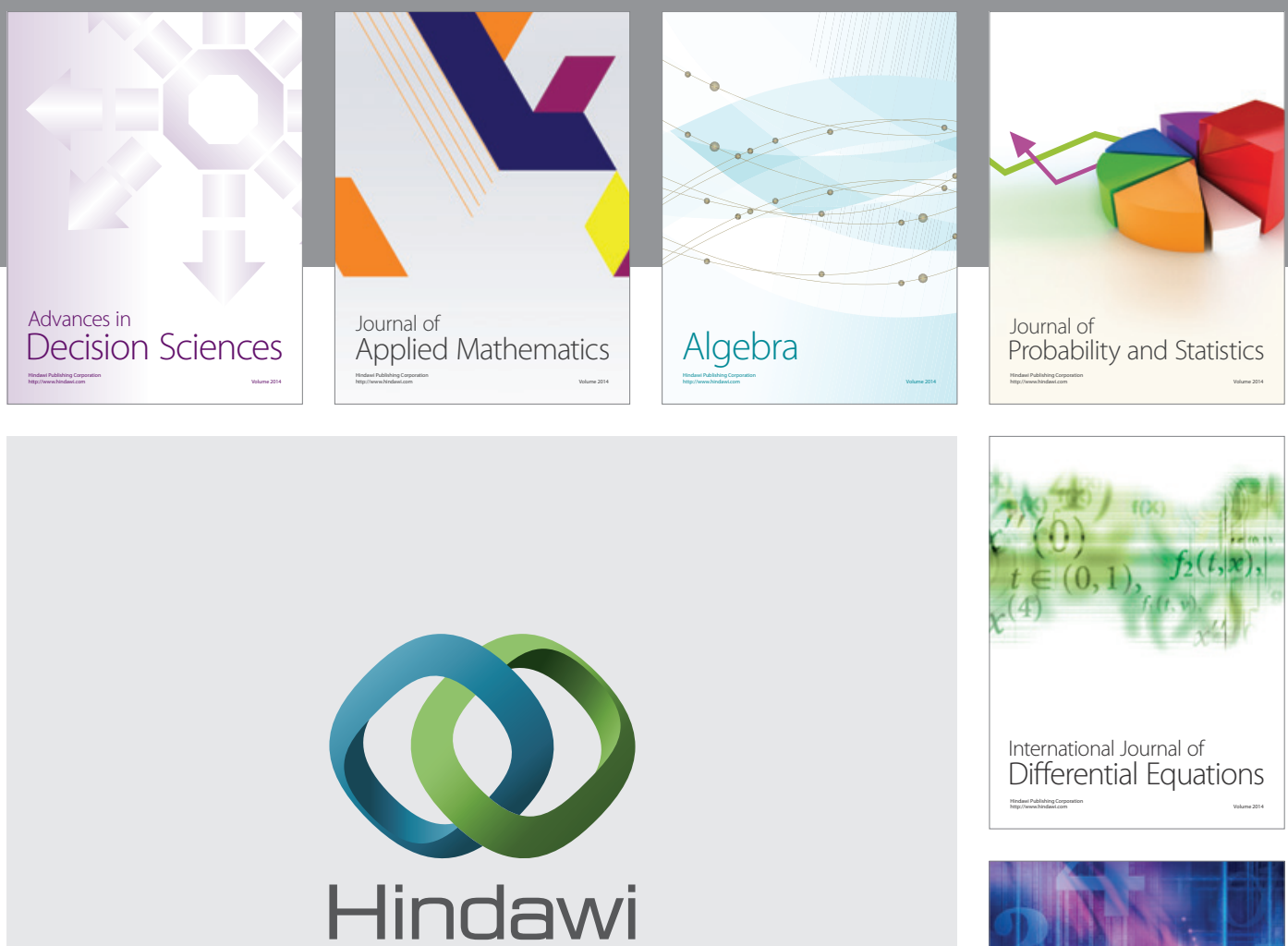

Submit your manuscripts at http://www.hindawi.com
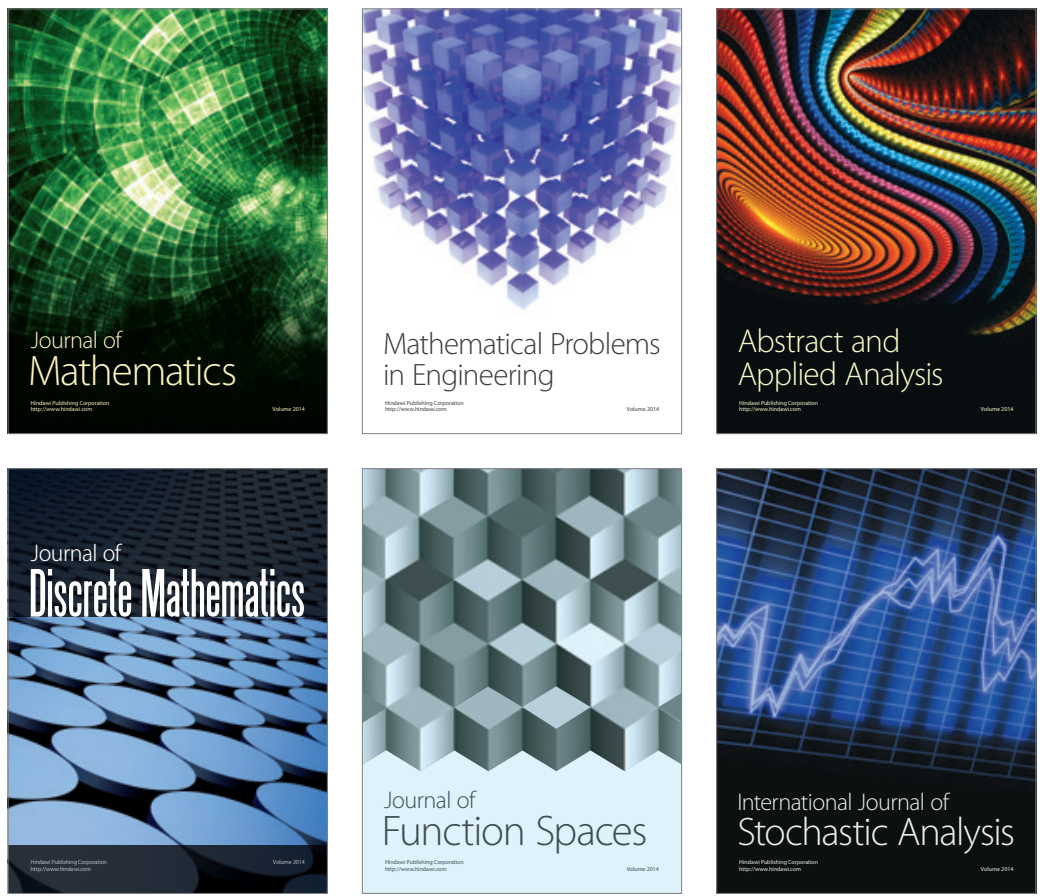

Journal of

Function Spaces

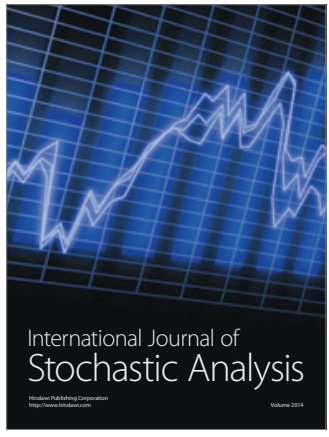

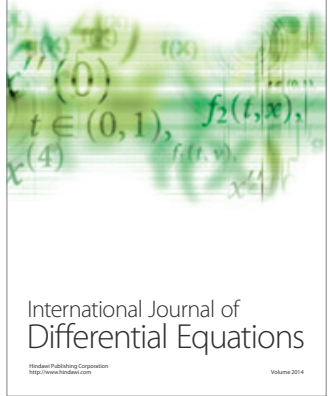
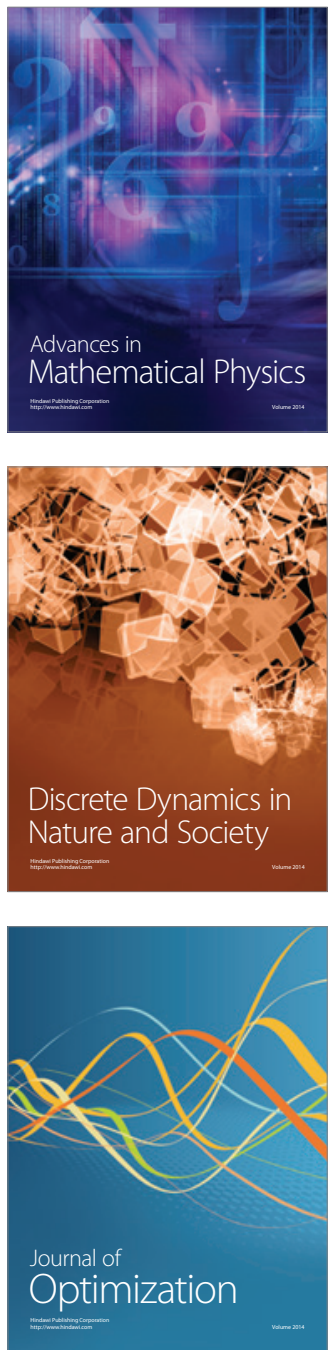